\title{
Cost-effective Surveillance for Infectious Diseases Through Specimen Pooling and Multiplex Assays
}

\author{
Christopher Bilder ${ }^{1}$, Joshua Tebbs ${ }^{2}$, Christopher McMahan ${ }^{3}$ \\ ${ }^{1}$ Statistics, University of Nebraska-Lincoln, Lincoln, Nebraska, United States, ${ }^{2}$ University of South Carolina, Columbia, South Carolina, United States, \\ ${ }^{3}$ Clemson University, Clemson, South Carolina, United States
}

Objective

To develop specimen pooling algorithms that reduce the number of tests needed to test individuals for infectious diseases with multiplex assays.

\section{Introduction}

An essential tool for infectious disease surveillance is to have a timely and cost-effective testing method. For this purpose, laboratories frequently use specimen pooling to assay high volumes of clinical specimens. The simplest pooling algorithm employs a two-stage process. In the first stage, a set number of specimens are amalgamated to form a "group" that is tested as if it were one specimen. If this group tests negatively, all individuals within the group are declared disease free. If this group tests positively, a second stage is implemented with retests performed on each individual. This testing algorithm is repeated across all individuals that need to be tested. In comparison to testing each individual specimen, large reductions in the number of tests occur when overall disease prevalence is small because most groups will test negatively.

Most pooling algorithms have been developed in the context of single-disease assays. New pooling algorithms are developed in the context of multiplex (multiple- disease) assays applied over two or three hierarchical stages. Individual risk information can be employed by these algorithms to increase testing efficiency.

\section{Methods}

Monte Carlo simulations are used to emulate pooling and testing processes. These simulations are based on retrospective chlamydia and gonorrhea testing data collected over a two-year period in Idaho, Iowa, and Oregon. For each simulation, the number of tests and measures of accuracy are recorded. All tests were originally performed by the Aptima Combo 2 Assay. Sensitivities and specificities for this assay are included in the simulation process. The $\mathrm{R}$ statistical software package is used to perform all simulations. For reproducibility of the research, programs are made available at www.chrisbilder.com/grouptesting to implement the simulations.

\section{Results}

Reductions in the number of tests were obtained for all states when compared to individual specimen testing. For example, the pooling of Idaho female specimens without taking into account individual risk information resulted in a $47 \%$ and a $51 \%$ reduction in tests when using two and three stages, respectively. With the addition of individual risk information, further reductions in tests occurred. For example, the pooling of Idaho female specimens resulted in an additional 5\% reduction of tests when compared directly to not using individual risk information. These reductions in tests were found to be related to the type of risk information available and the variability in risk levels. For example, males were found to have much more variability than females. For Idaho, this resulted in a $15 \%$ further reduction in tests than when not using the risk information.

\section{Conclusions}

Significant reductions in the number of tests occur through pooling. These reductions are the most significant when individual risk information is taken into account by the pooling algorithm. 


\section{Acknowledgement}

This research is supported by Grant R01 AI121351 from the National Institutes of Health. The authors thank Cardea Services and the state public health laboratories in Idaho, Iowa, and Oregon for providing access to their testing data. 